\title{
Scaling the Non-linear Impact Response of Flat and Curved Composite Panels
}

\author{
Damodar R. Ambur* \\ NASA Glenn Research Center, Cleveland, $\mathrm{OH} 44135$ \\ Prasad B. Chunchu, ${ }^{\dagger}$ Cheryl A. Rose, ${ }^{\dagger}$ Paolo Feraboli, ${ }^{\S}$ Wade C. Jackson ${ }^{* *}$ \\ NASA Langley Research Center, Hampton, VA 23681
}

\begin{abstract}
The application of scaling laws to thin flat and curved composite panels exhibiting nonlinear response when subjected to low-velocity transverse impact is investigated. Previous research has shown that the elastic impact response of structural configurations exhibiting geometrically linear response can be effectively scaled. In the present paper, a preliminary experimental study is presented to assess the applicability of the scaling laws to structural configurations exhibiting geometrically nonlinear deformations. The effect of damage on the scalability of the structural response characteristics, and the effect of scale on damage development are also investigated. Damage is evaluated using conventional methods including C-scan, specimen de-plying and visual inspection of the impacted panels. Coefficient of restitution and normalized contact duration are also used to assess the extent of damage. The results confirm the validity of the scaling parameters for elastic impacts. However, for the panels considered in the study, the extent and manifestation of damage do not scale according to the scaling laws. Furthermore, the results indicate that even though the damage does not scale, the overall panel response characteristics, as indicated by contact force profiles, do scale for some levels of damage.
\end{abstract}

\section{Introduction}

Composite materials are increasingly being used in high performance aerospace structures where both stiffnessto-weight and strength-to-weight are important. The general susceptibility of composite materials to impact damage, however, is a concern that limits their potential. Test data show that the compression strength of composite structures can be reduced by impact damage, even if the damage is not detectable by visual inspection. Further, the impact problem of composite structures is complex as it involves complicated structural response, localized out-outof-plane loading, possible strain-rate effects, and interaction of several failure modes. Also, several parameters influence the impact response and damage in composite structures and include impactor mass, size and speed; specimen or target geometry, materials, laminate sequence, and boundary or support conditions; and the relative magnitudes of impactor and target masses [1-5].

In order to develop damage tolerant design criteria for composite structures, impact testing for material characterization is needed. In accordance with the Building Block Approach to the design of composite structures, information for the design process is gained by gathering an extensive material database on progressively more complex structural configurations, without having to invest a large amount of capital for testing a large number of proto-type full scale elements. However, the results obtained from small, laboratory scale tests cannot always be extrapolated to predict the response of large structures made from the same material because of potential size effects. While this is true in many aspects of aircraft design, it is especially true in the area of foreign object damage (FOD) resistance and damage tolerance, where the complicated nature of the loading and of the failure mechanisms hinders the understanding of the governing phenomena. In the case of high-velocity impact events, such as hail ice

\footnotetext{
${ }^{*}$ Chief, Structures and Acoustics Division. Associate Fellow, AIAA.

${ }^{\dagger}$ Research Engineer, Eagle Aeronautics. Member, AIAA.

* Senior Research Engineer, Mechanics of Structures and Materials Branch. Senior Member AIAA.

${ }^{\S}$ Research Contractor, University of California. Member AIAA.

** Aerospace Engineer, Army Research Laboratories.
} 
or runway debris impacts, where the total contact duration of the impact event is of the same order of magnitude as the flexural waves traveling in the specimen, the dynamic response of the target is highly localized and, generally, laboratory scale configurations can be employed to simulate the response of larger and more complex structures [4]. In the case of low-velocity impact events, such as tool drops, the contact duration of the event is much longer, and the global structural response characteristics of the target structure are more significant [5].

Many studies can be found in the literature which investigate the impact response of different impactor/target combinations. Traditional parametric investigations have been conducted to isolate the influence of a specific structural parameter on the measured impact response, while keeping all other parameters unchanged [6-11]. Among these characteristic parameters, the most frequent for the impactor are mass, velocity, material, shape and size, while for the target laminate they are aperture size, laminate thickness, material, curvature and boundary conditions. On the other hand, very little research has been focused toward the understanding of the scaling behavior of transversely loaded plates, either in a static or impact condition [12-15]. The majority of the work has focused on the development and assessment of scaling laws for the elastic, small deformation impact response of composite plates. Morton [16] verified the use of non-dimensional analysis for predicting the elastic (undamaged) behavior of carbon/epoxy beams, subjected to impact in a three-point bending configuration. He also pointed out important size effects on strength, indicating that smaller specimens exhibited higher strength than smaller specimens. Qian et al. [12] derived scaling laws for transversely impacted plates, using an analysis based on the geometrically linear dynamic plate equations, and showed that the impact response of plates undergoing linear deformations can be adequately scaled, provided no damage develops in the specimen. Swanson et al. experimentally verified that the undamaged response of cylinders to impact can also be scaled [17]. Swanson also conducted a preliminary experimental study to include the effect of size on the formation of damage in impacted plates [18]. Based on his results and fracture mechanics, he concluded that there is an apparent dependence of delamination area on absolute size. Morton suggested, however, that the total damage is related to the energy absorbed by the composite, not just the delamination area, and methods are needed to measure and quantify the total damage [16].

Previous analytical and experimental studies [7-11] have shown that thin composite panels, representative of composite fuselage shell structures, exhibit geometrically nonlinear response when impacted at very low impact energies. Therefore, geometrically nonlinear effects must be considered when developing scaling laws for the impact response of these structures. The results presented in the literature reviewed herein indicate that while the scalability of the elastic impact response of composite plates exhibiting geometrically linear response is well established, little effort has been directed to developing and assessing scaling laws for geometrically nonlinear impact response. In addition, the effect of impact damage on the scalability of the structural response characteristics, and the effect of scale on damage development are not well understood. Therefore, the first objective of the present paper is to demonstrate, analytically and experimentally, that the geometrically nonlinear elastic impact response of flat and curved composite panels can be accurately scaled. The assessment is then extended to include damage-inducing impacts. Assessment of the scalability of the structural response is made by comparison of contact force and strain profiles for two sizes of flat and curved panels that have been geometrically scaled. The effect of scale on damage development is then addressed. The extent of damage is characterized by means of traditional methods (i.e. ultrasonic C-scans and visual inspection) and less conventional techniques (i.e. post-failure elastic impact testing [19]), and an attempt is made to obtain a quantitative and qualitative correlation between the damage states within scaled specimens. These scaling laws for flat and curved anisotropic composite panels are needed to extend the applicability of laboratory impact data to complex aerospace structures, such as fuselage panels, while minimizing the testing activities related with the determination of compression-after-impact (CAI) strength for each structural configuration.

\section{Nonlinear Analysis and Scaling Rules}

Determining the scaling behavior of the impact response of composite panels requires the application of the same scaling factor to all quantities that participate in the event. Therefore, if the target's in-plane and transverse dimensions are scaled by a factor $\lambda$, the diameter and the weight of the impactor (hence the impact energy), and the radius of curvature of the panel need to be scaled accordingly. Previous investigators have obtained rules for scaling the elastic impact response of composite plates by considering the differential equations governing the dynamic, geometrically linear response of an orthotropic plate $[12,13]$. These studies showed that when the impact velocity is equal for the reference and scaled configurations, and the geometric characteristics of the target and impactor scale by $\lambda$, time scales by $\lambda$, and the impactor weight scales according to $\lambda^{3}$. Impact energy also scales by $\lambda^{3}$. The maximum strain magnitudes in the reference and scaled configurations are equal. Swanson et al. extended the 
scaling investigation to the impact response of composite cylinders, and found that the cylinder radius is a geometric factor, and scales by $\lambda[17]$.

Previous analytical and experimental studies [6-11] have shown that thin composite panels exhibit geometrically nonlinear response when impacted at very low impact energies. Thin flat panels exhibit a nonlinear stiffening response, and some thin curved panels exhibit an initial nonlinear softening response. Therefore, scaling laws and analysis methods for these structures must include the effects of geometric nonlinearity. A geometrically nonlinear analysis method has been previously developed for predicting the elastic impact response of thin, curved, anisotropic panels [6-9]. The analysis method is based on the Lagrangian equations of motion, which are solved using the Rayleigh-Ritz method and assumed mode shapes. Generalized beam functions are used to represent the modes, so the analysis is general with respect to boundary conditions [20]. The system of equations is solved using the standard fourth order Runge-Kutta method. Results obtained from the nonlinear analysis code are used to verify that the scaling laws developed for small deformations apply to the thin composite panels studied in the present investigation. Further, the results show that the radius of a curved panel scales by $\lambda$. A summary of the scaling parameters used in the present study is provided in Table 1.

\section{Test Specimens, Test Apparatus and Tests}

Six flat and cylindrically curved composite panels were considered in the present study, and are summarized in Table 2. Analyses and experiments were conducted for flat and curved panels of two sizes that were geometrically scaled. The reference configuration, $\lambda=1$, is a quasi-isotropic $[45 / 0 /-45 / 90]_{\mathrm{s}}$ laminate with a 5.0 -inch-square test section. The reference configuration curved panel has a radius of $15 \mathrm{in}$. The scaled configurations, $\lambda=2$, have a 10.0-inch-square test section, and for the curved panels the radius is 30 in. Scaled thicknesses are obtained using both ply-level and sub-laminate scaling [14,16]. For ply-level scaling, the scaling operation is performed by multiplying the effective ply thickness by $\lambda$, and the scaled 16 -ply laminate is $\left[45_{2} / 0_{2} /-45_{2} / 90_{2}\right]_{\mathrm{s}}$. With the ply-level scaling approach complete similitude is obtained, but conventional damage-tolerant design guidelines discourage the clumping of plies of the same orientation. Sub-laminate scaling, on the other hand, is potentially more practical, and is obtained by multiplying by $\lambda$ the entire repetitive unit of the stacking sequence. The sub-laminate scaled 16-ply laminate is $[45 / 0 /-45 / 90]_{2 s}$. With this scaling approach, similitude in bending stiffnesses is not maintained.

The specimens were fabricated from graphite-epoxy pre-impregnated unidirectional tape material made by Hercules, Inc. AS4/3501-6 and AS4/3502 material systems were used. The AS4/3501-6 material system was used to fabricate all of the specimens except for the sub-laminate-scaled curved panels (Panels C4). The mechanical properties for the AS4/3502 graphite-epoxy material system are as follows: longitudinal modulus $E_{1}=20.0 \mathrm{Msi}$, transverse modulus $E_{2}=1.60 \mathrm{Msi}$, in-plane shear modulus $G_{12}=0.88 \mathrm{Msi}$, and major Poisson's ratio $v_{12}=0.31$.

Table 1. Summary of Scaling Parameters

\begin{tabular}{c|c}
\hline Parameter & $\begin{array}{c}\text { Scaling } \\
\text { Factor }\end{array}$ \\
\hline Support Span & $\lambda$ \\
Laminate Thickness & $\lambda$ \\
Impactor Diameter & $\lambda$ \\
Curvature & $\lambda$ \\
Impactor Mass & $\lambda^{3}$ \\
Impact Energy & $\lambda^{3}$ \\
Contact Duration & $\lambda$ \\
Displacement & $\lambda$ \\
Peak Force & $\lambda^{2}$ \\
Damage Area & $\lambda^{2}$ \\
\hline
\end{tabular}


Table 2. Summary of Panel Configurations

\begin{tabular}{c|cccccccc}
\hline Config & $\begin{array}{c}\text { Scaling } \\
\text { Factor } \\
\lambda\end{array}$ & $\begin{array}{c}\text { Aperture } \\
\text { size } \\
\text { D, in. }\end{array}$ & $\begin{array}{c}\text { Radius } \\
\text { Curvature } \\
\text { R, in. }\end{array}$ & $\begin{array}{c}\text { Avg. } \\
\text { thick. } \\
\text { T, in. }\end{array}$ & $\begin{array}{c}\text { No. } \\
\text { Plies }\end{array}$ & $\begin{array}{c}\text { Laminate } \\
\text { Sequence }\end{array}$ & $\begin{array}{c}\text { Impactor } \\
\text { Diameter } \\
\text { D, in. }\end{array}$ & $\begin{array}{c}\text { Impactor } \\
\text { Mass } \\
\text { W, lb }\end{array}$ \\
\hline C1 & 1 & $5 \times 5$ & FLAT & 0.0546 & 8 & {$[45 / 0 /-45 / 90]_{\mathrm{s}}$} & 0.5 & 2.78 \\
C2 & 1 & $5 \times 5$ & 15 & 0.0514 & 8 & {$[45 / 0 /-45 / 90]_{\mathrm{s}}$} & 0.5 & 2.78 \\
$\mathrm{C} 3$ & 2 & $10 \times 10$ & FLAT & 0.1071 & 16 & {$[45 / 0 /-45 / 90]_{2 \mathrm{~s}}$} & 1.0 & 22.46 \\
$\mathrm{C} 4$ & 2 & $10 \times 10$ & 30 & 0.0979 & 16 & {$[45 / 0 /-45 / 90]_{2 \mathrm{~s}}$} & 1.0 & 22.46 \\
$\mathrm{C} 5$ & 2 & $10 \times 10$ & FLAT & 0.1035 & 16 & {$\left[45_{2} / 0_{2} /-45_{2} / 90_{2}\right]_{\mathrm{s}}$} & 1.0 & 22.46 \\
$\mathrm{C} 6$ & 2 & $10 \times 10$ & 30 & 0.0980 & 16 & {$\left[45_{2} / 0_{2} /-45_{2} / 90_{2}\right]_{\mathrm{s}}$} & 1.0 & 22.46 \\
\hline
\end{tabular}

The mechanical properties for the AS4/3501-6 graphite-epoxy material system are as follows: $E_{1}=21.0$ Msi, transverse modulus $E_{2}=1.49 \mathrm{Msi}$, in-plane shear modulus $G_{12}=0.99 \mathrm{Msi}$, and major Poisson's ratio $v_{12}=0.30$. Unidirectional tapes were laid up on an aluminum tool and cured in an autoclave using the manufacturer's recommended procedures to form the base and scaled quasi-isotropic panels. The resulting panels were ultrasonically inspected to establish specimen quality, and then machined to size.

\section{Test Apparatus and Tests}

The specimens were clamped in fixtures described in Ref. 9 and shown in Fig. 1. The square aperture has edge dimensions equal to 5 inches for the reference configuration and equal to 10 inches for the scaled configurations $(\lambda=2)$. The clamping screws that attach the fixture base-plate and face-plate penetrate the test specimen through holes drilled in the specimen. This fixture has been used in previous investigations [6-9], and was designed to minimize translations and rotations at the test section boundary. Satisfying these conditions is particularly important for studying the impact response of thin plates and curved panels in which geometrically nonlinear effects are significant.

Three sets of panels were tested in this investigation. As shown in Table 2, each set consists of six panels. Static indentation tests were conducted on one set of panels, prior to dropped-weight impact testing. Low-velocity impact is considered to be a quasi-static event and static indentation tests can provide useful information for interpreting a structure's response to low-speed impact $[19,21]$. The static indentation tests were conducted using the same fixtures and indentors used for the impact testing. Static indentation load-displacement curves obtained for the flat and curved panels are shown in Figs. 2a and b, respectively. The critical force, defined as the value of the force at

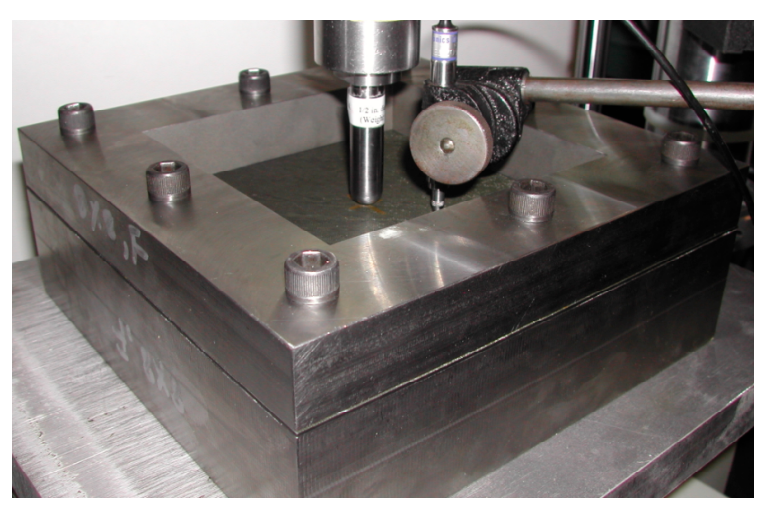

(a) Static indentation testing set-up

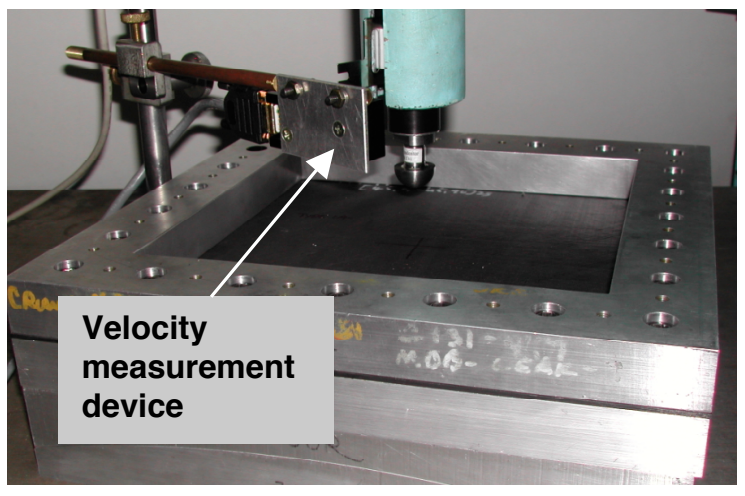

(b) Dropped-weight impact testing set-up

Figure 1. Static indentation and dropped-weight impact testing set-up. 
the onset of failure, corresponds to the first significant drop in the loading curve. Additional drops in the loading curve correspond to additional failures. The results in Fig. 2 show that there are no drops in the load-displacement curves for the flat and curved 8-ply laminates prior to reaching the ultimate load, even though audible cracks were reported early during the testing. For the sub-laminate scaled 16-ply laminate, on the other hand, there is a visible drop in load early in the loading, and for all of the scaled-up laminates ultimate load is preceded by at least one sharp drop in the load-deflection curve. The corresponding static deformation energy curves, obtained by numerically integrating the load-displacement data are provided in Fig. 3. Critical energy is defined as the value of the impact energy corresponding to the critical force. Using this relationship and the data from Fig. 2, impact energies were defined for the dropped-weight impact testing for elastic response and for induced damage. For the small panels, elastic tests were performed at a nominal energy level of $0.35 \mathrm{ft}-\mathrm{lb}$. The elastic impact tests on the scaled panels were performed at a nominal impact energy level of $2.8 \mathrm{ft}-\mathrm{lb}$, using the higher scaled mass, and keeping the velocity the same as the velocity for the reference panels. Similarly, damage-inducing impact tests were conducted at nominal 1.125 and $2 \mathrm{ft}-\mathrm{lb}$ energy levels for the reference panels, and at nominal 9 and $16 \mathrm{ft}-\mathrm{lb}$ energy levels for the scaled panels. These impact energy levels were chosen so that damage would develop in all of the panel configurations.

Dropped-weight impact tests were conducted on the remaining two sets of panels. In both sets, three impact tests were conducted on each of the six panels. The first test was conducted on each panel to determine the elastic impact response of the pristine panel. The second test was conducted at one of the damage-inducing energies. The third test was conducted on the damaged panel at the same elastic impact energy as in the first test to determine the elastic impact response of the damaged panel. The ratio of damaged to pristine structural stiffness, as measured by the ratio of total contact duration of the elastic impact event for the pristine and damaged panels, is thought to be indicative of the extent of damage within the laminate and its residual stiffness. The coefficient of restitution (COR), a measure of damage resistance, is defined as the ratio of rebound to impact velocity. For configurations exhibiting geometrically linear response to impact, COR and contact duration have been shown to provide information on the instantaneous stiffness of an impact-damaged structure [19]. Contact duration and coefficient of restitution of the different configurations are compared in the present study to try and extend the use of these quantities for assessing the state of damage in structures exhibiting geometrically nonlinear response.

Each specimen was impacted on one side of the specimen at the specimen center by a dropped-weight impactor. As summarized in Table 2, the dropped-weight assembly weighed $2.78 \mathrm{lb}(\lambda=1)$ and $22.46 \mathrm{lb}(\lambda=2)$ and included a force transducer and a 0.5 - and 1.0-in.-diam hemispherical steel tup for $\lambda=1,2$, respectively. All specimens were instrumented with a single, strain gage on the specimen surface opposite to the side to be impacted. The gage was aligned with the panel longitudinal axis and was located 0.5 and 1.0 -inches from the specimen center along the panel

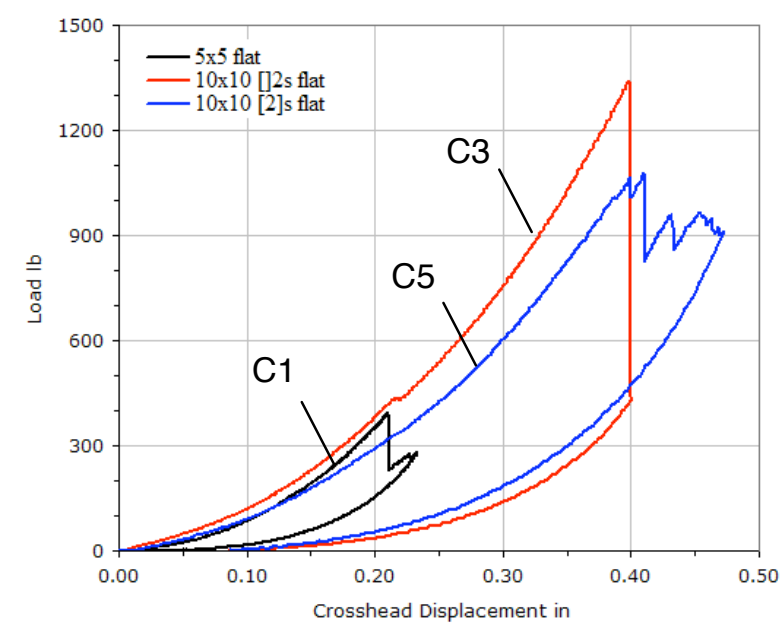

(a) Flat Panels

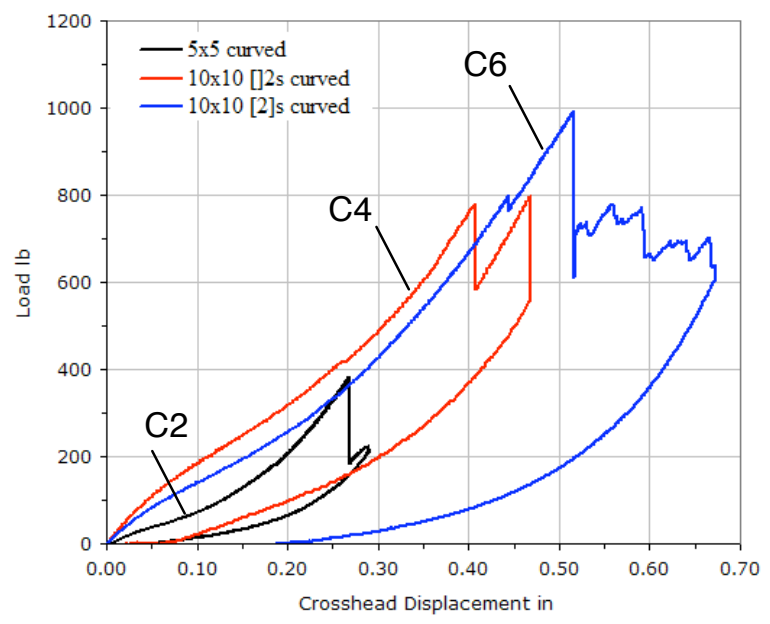

(b) Curved Panels

Figure 2. Force-displacement response from static indentation tests of the flat and curved panel configurations. 


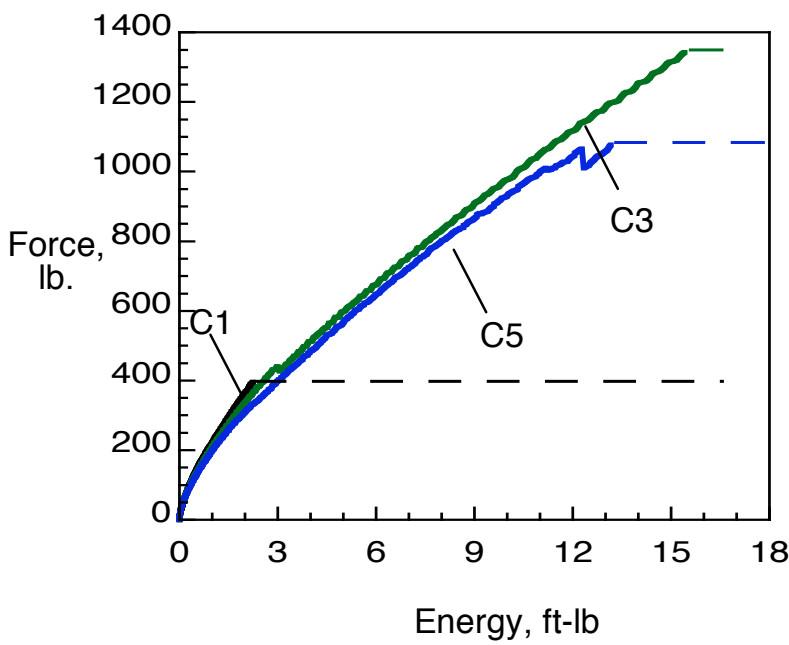

(a) Flat panels

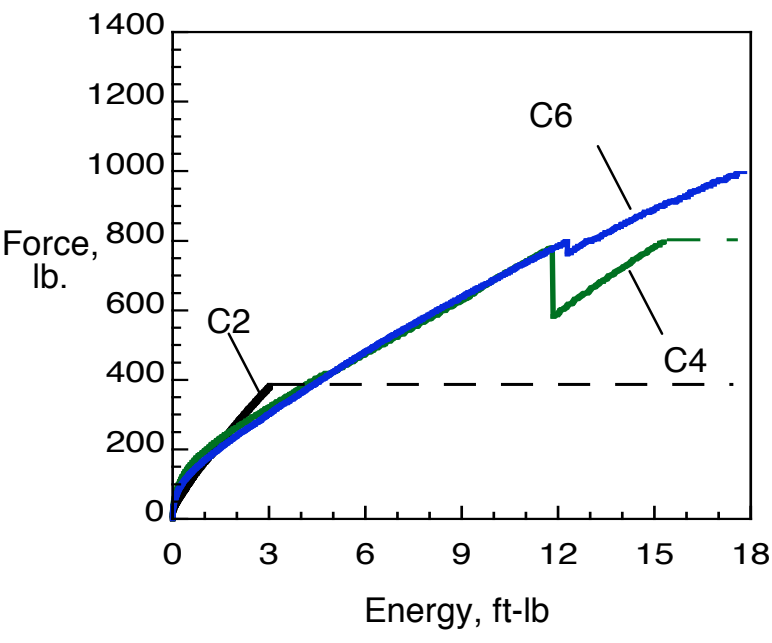

(b) Curved panels

Figure 3. Force-energy curves from static indentation tests of the flat and curved panel configurations.

longitudinal axes for the $\lambda=1,2$ configurations, respectively. A velocity-measuring device (see Fig. 1) was installed to measure the impactor velocity just prior to specimen contact. All data were recorded using a digital oscilloscope.

\section{Results}

Numerically predicted and experimentally measured results for the six panel configurations shown in Table 2 subjected to low-velocity dropped-weight impact are presented in this section. First, numerical predictions are presented for an elastic impact to demonstrate that the scaling laws apply to flat and curved panels exhibiting geometrically nonlinear response, and the panel radius is a geometric parameter that scales by $\lambda$. Experimental results are then presented for both the elastic and damage-inducing impact response of the flat and curved panels to validate the scaling laws and to assess the scale effect on damage development. Elastic impact tests were performed at a nominal impact energy level of $0.35 \mathrm{ft}-\mathrm{lb}$ for the reference panels, and at a nominal impact energy level of 2.8 $\mathrm{ft}-\mathrm{lb}$ for the scaled panels. Damage-inducing tests were performed at nominal $1.125 \mathrm{ft}-\mathrm{lb}$ and $2 \mathrm{ft}-\mathrm{lb}$ impact energy levels for the reference panels, and at nominal $9 \mathrm{ft}-\mathrm{lb}$ and $16 \mathrm{ft}-\mathrm{lb}$ impact energy levels for the scaled panels. Force vs. time and strain vs. time plots are presented and compared for the different configurations to provide an assessment of the scalability of the structural response of panels with and without damage. Damage development is assessed by comparing critical and peak force values, coefficient of restitution and contact duration for the different configurations, C-scan images and fiber damage areas observed in de-plied specimens.

\section{Numerical Predictions}

Representative nonlinear analysis results for the contact force and axial strain profiles at the experimental gage location for the curved panel configurations impacted at the center of the panel are shown in Figs. 4 and 5. All sides of the panel were clamped, and the panels were impacted at $0.35 \mathrm{ft}-\mathrm{lb}$ and $2.8 \mathrm{ft}-\mathrm{lb}$ for the reference and scaled-up configurations, respectively. This impact energy corresponds to the nominal elastic impact energy that was used in the experimental investigations. Curves are shown in Fig. 4 for the un-scaled and scaled force response predictions as a function of time. The scaled response curves for the scaled-up configurations were obtained by scaling the contact force by $1 / \lambda^{2}$, and time by $1 / \lambda$. The results in Fig. 4 show that the scaled contact force profiles for the scaled-up configurations nearly collapse to the contact force profile for the reference configuration. A small difference is seen between the predictions for the reference configuration and for the sub-laminate scaled laminate because of the lack of bending stiffness similitude that was mentioned earlier. Similar results are obtained for the axial strain, as shown in Fig. 5. The un-scaled axial strain profiles show that the peak strain magnitude is equal for the reference and scaled-up configurations. Multiplying the time scale for the scaled-up configurations by $1 / \lambda$ in accordance with the time scaling above reduces all of the response predictions to a single curve. 


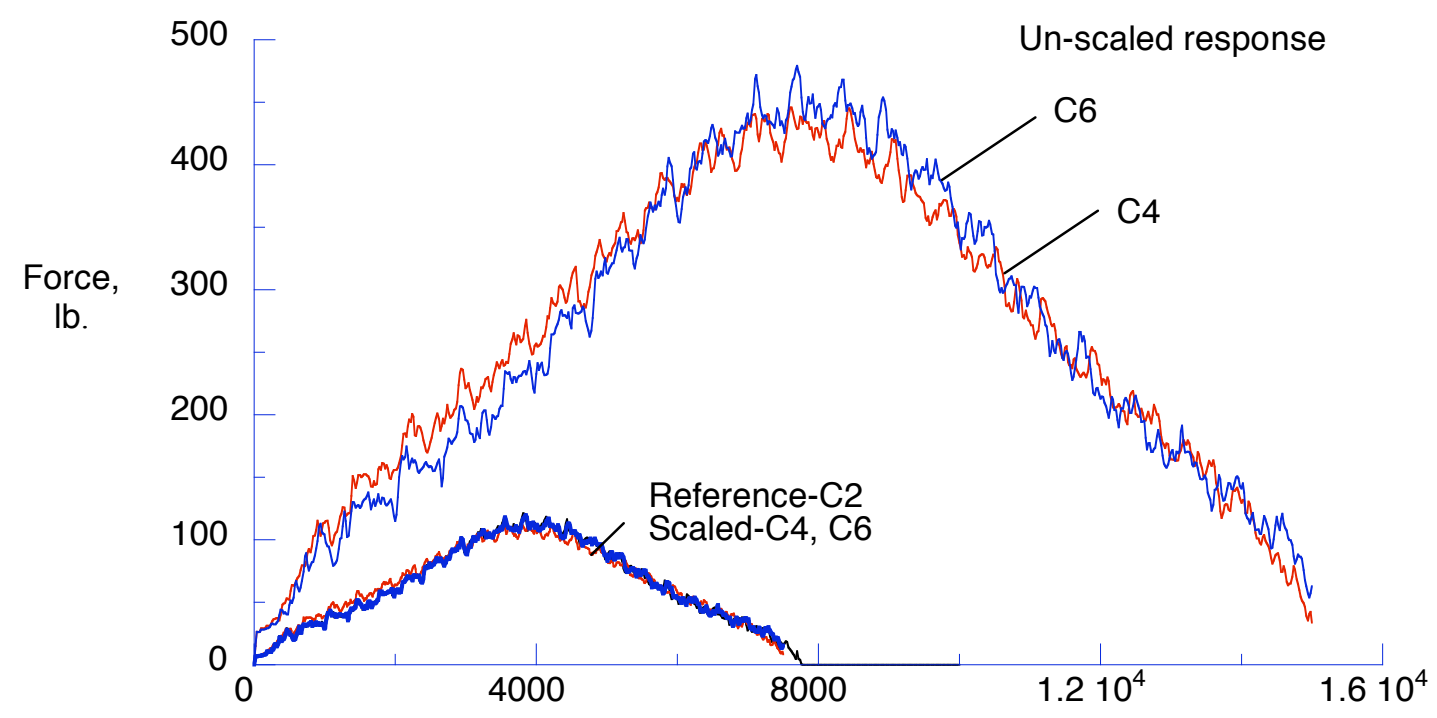

Time, $\mu \mathrm{sec}$

Figure 4. Analytical contact force profiles for the curved panel configurations centrally impacted at $0.35 \mathrm{ft}-\mathrm{lb}$ and $2.8 \mathrm{ft}-\mathrm{lb}$ for the reference and scaled-up configurations, respectively.

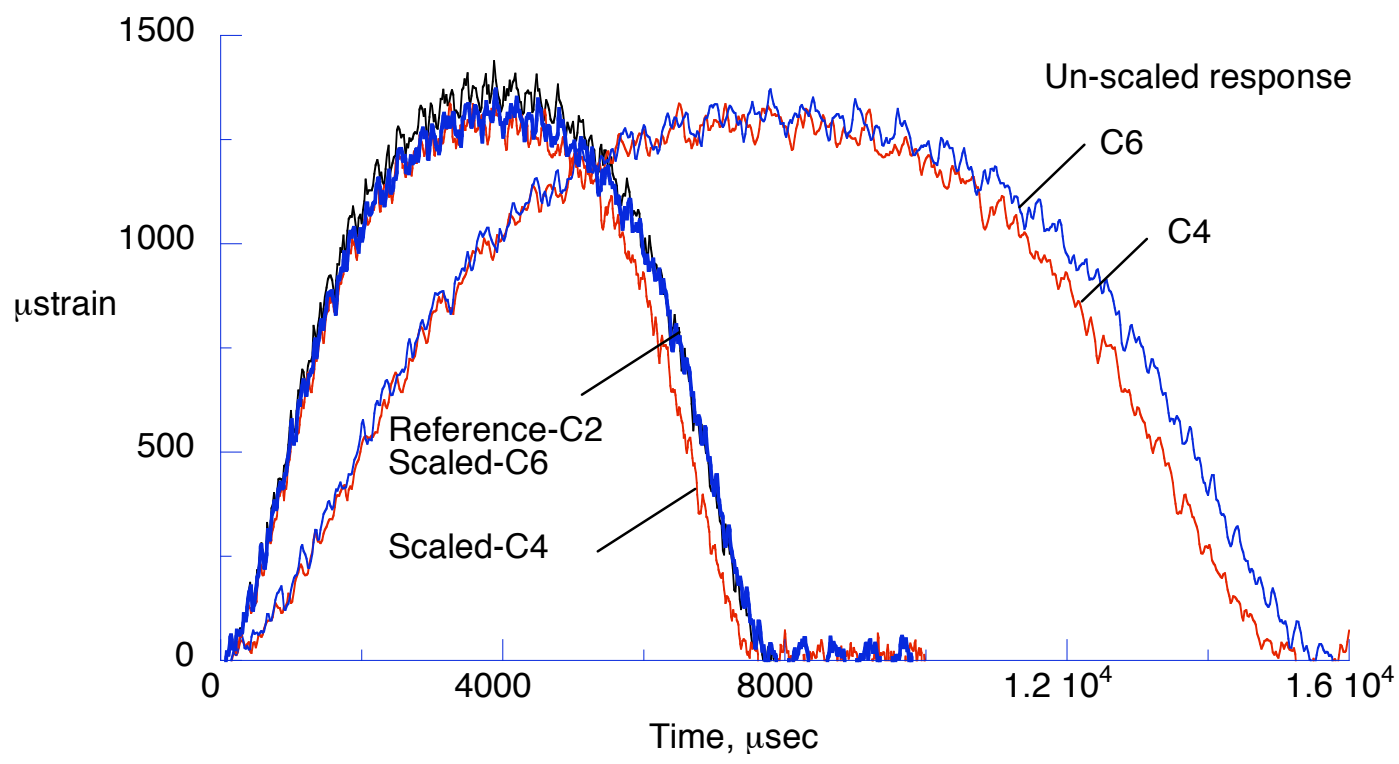

Figure 5. Analytical axial strain profiles at 0.5 in and 1.0 from the point of impact and on back surface for the curved panel configurations centrally impacted at $0.35 \mathrm{ft}-\mathrm{lb}$ and $2.8 \mathrm{ft}-\mathrm{lb}$ impact energy for the reference and scaled-up configurations, respectively. 


\section{Experimental Results - Elastic Impact}

Experimental contact force profiles for the pristine flat panels subjected to the elastic dropped-weight impact are shown in Fig. 6. Curves in Fig. 6 and in subsequent figures are labeled using the configuration numbers provided in Table 2. Curves are shown in Fig. 6a for the measured and scaled force response predictions as a function of time. The scaled response curves for the scaled-up configurations were obtained by scaling the contact force by $1 / \lambda^{2}$, and time by $1 / \lambda$. The results in Fig. 6 show that the scaled contact force profiles for the scaled-up configurations nearly collapse to the contact force profile for the reference configuration. The primary source of difference between the results shown for the reference and scaled configurations is the difference in measured impact velocity for the three configurations. Precisely maintaining a consistent drop height between tests, and hence obtaining consistent impact velocity is difficult. The effect of different impact velocity between tests is the most significant for the lower energy elastic impact tests. The curves shown in Fig. $6 \mathrm{~b}$ have been normalized and adjusted to account for the difference in measured impact velocity between the reference and scaled-up configurations. Forces are normalized by the peak force obtained for the reference configuration, $F_{\text {MaxR }}$, multiplied by $\lambda^{2}$. To make the adjustment in force, the measured impact energy from the reference configuration test, $E_{R}$, is multiplied by $\lambda^{3}$ to obtain the target scaled impact energy, $\lambda^{3} E_{R}$. Using this value of energy and the measured energy from the scaled-up configuration test, $E_{s}$, a ratio of forces is obtained from the static indentation force vs. energy curve, shown in Fig. 3, for the scaled-up configuration. The forces on the curve correspond to the scaled energy, $\lambda^{3} E_{R}$, and the measured energy, $E_{s}$, and are denoted as $F_{\lambda E_{R}}^{S I}$ and $F_{E_{S}}^{S I}$, respectively. The normalized and adjusted force is determined by

$$
\bar{F}=\left(\frac{F(t)}{F_{\text {MaxR }} \lambda^{2}}\right)\left(\frac{F_{\lambda^{3} E_{R}}^{S I}}{F_{E_{S}}^{S I}}\right)
$$

where $F_{\text {MaxS }}$ is determined from the static-indentation force vs. energy curve and is the force corresponding to the actual impact energy for the scaled-up configuration. Strain values are adjusted in the same manner. Contact duration is independent of impact velocity for elastic impacts of structures exhibiting geometrically linear response. The change in contact duration with change in impact velocity is negligible for the higher energy impacts, so time was not adjusted. Time is normalized by the contact duration for the reference configuration, $\mathrm{C}_{\mathrm{TR}}$, multiplied by $\lambda$; that is

$$
\bar{t}=t / \lambda C_{T R}
$$

where $\lambda=1$, and 2 for the reference and scaled configurations, respectively.

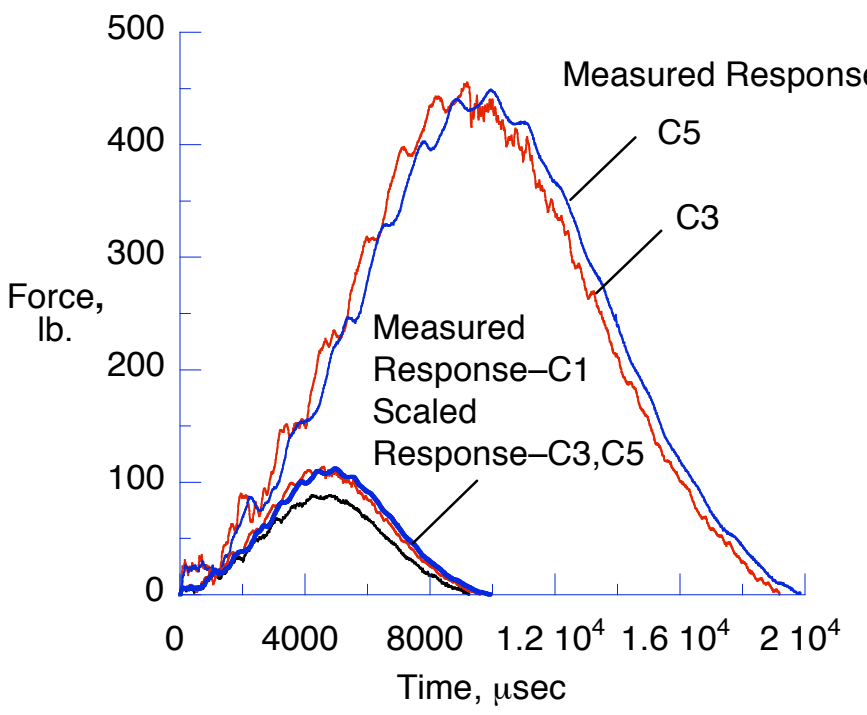

(a) Un-scaled and scaled contact force profiles

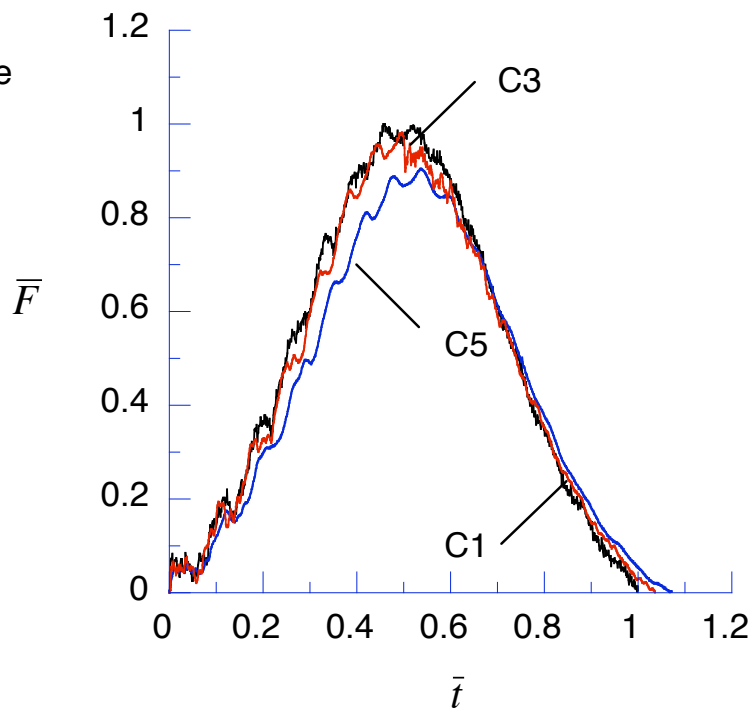

(b) Normalized and adjusted contact force profiles

Figure 6. Experimental contact force profiles for the flat panel configurations centrally impacted at nominal $0.35 \mathrm{ft}-\mathrm{lb}$ and $2.8 \mathrm{ft}-\mathrm{lb}$ impact energy for the reference and scaled-up configurations, respectively. 
Results are shown for the back surface strains in Fig. 7. The results shown in Fig. 7 indicate that the contact durations for the strain profiles scale very well using the parameters defined in Table 1 for the flat panel configurations. The larger strain response for the reference panel compared to the strain response for the scaled-up configurations is possibly caused by the impactor striking the panel slightly off center, and closer to the gage. Similar results for the curved panel configurations are shown in Fig. 8, and confirm that the radius is a geometric factor that scales by $\lambda$.

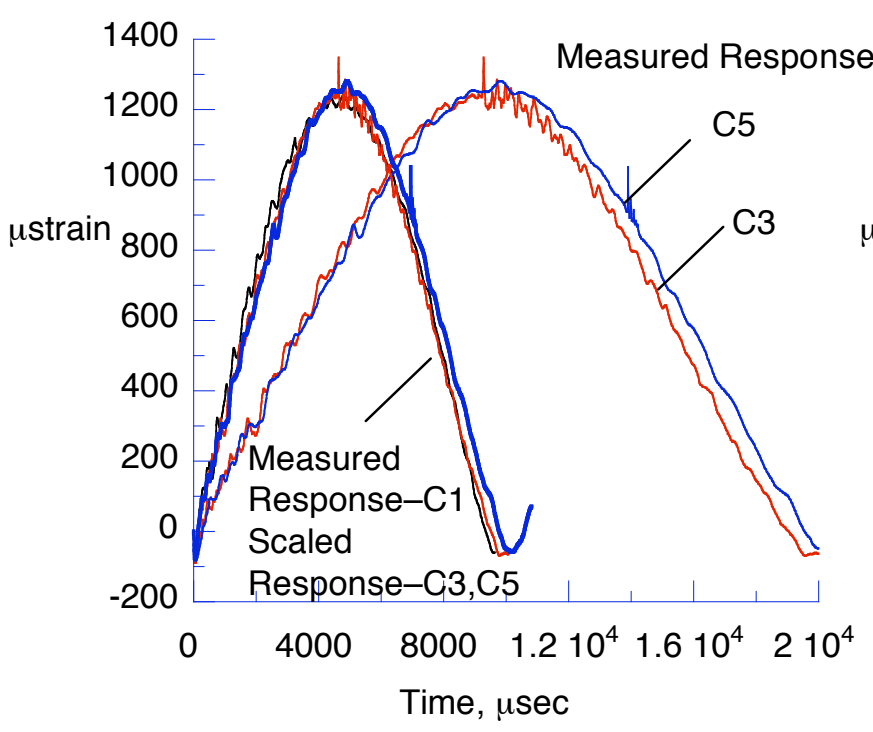

(a) Un-scaled and scaled axial strain profiles

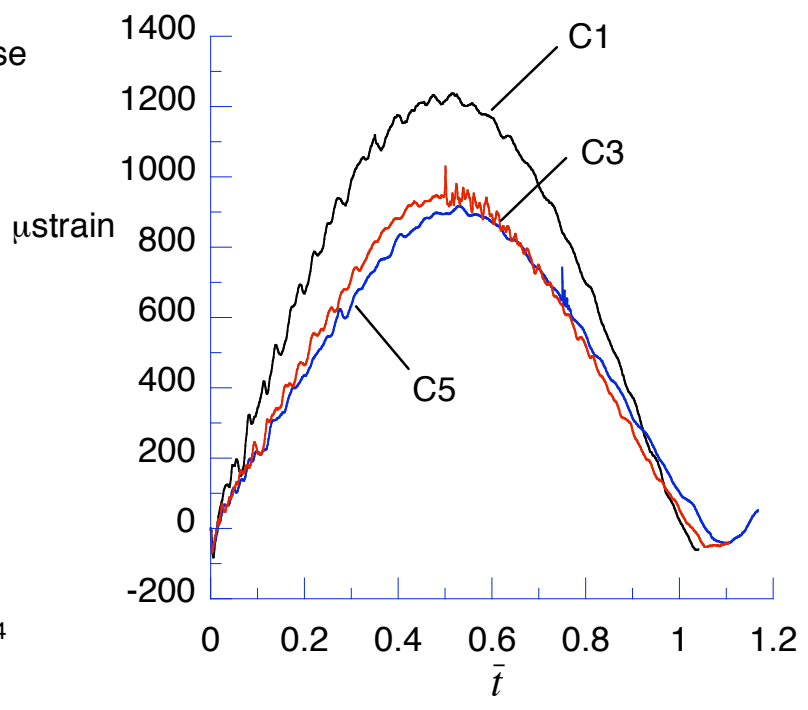

(b) Normalized and adjusted axial strain profiles

Figure 7. Experimental axial strain profiles for the flat panel configurations centrally impacted at nominal $0.35 \mathrm{ft}-\mathrm{lb}$ and $2.8 \mathrm{ft}-\mathrm{lb}$ impact energy for the reference and scaled-up configurations, respectively.

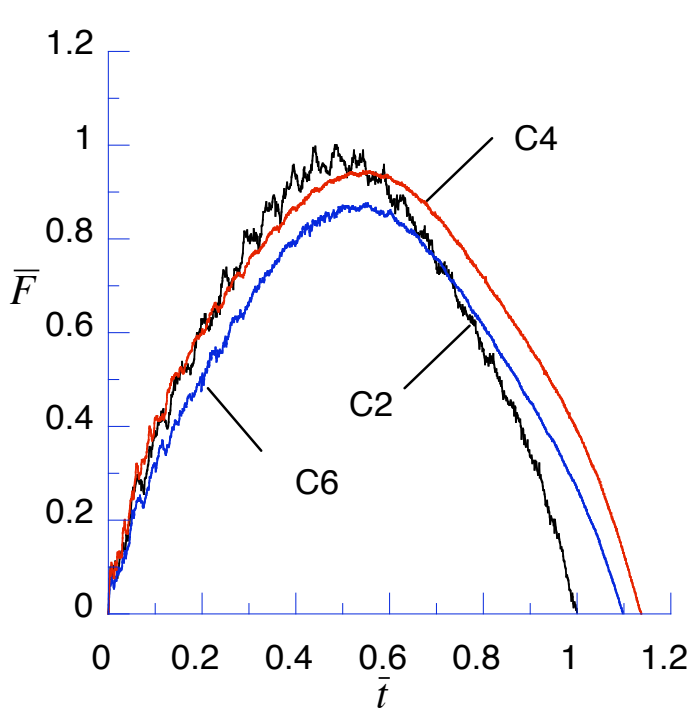

(a) Normalized and adjusted contact force profiles

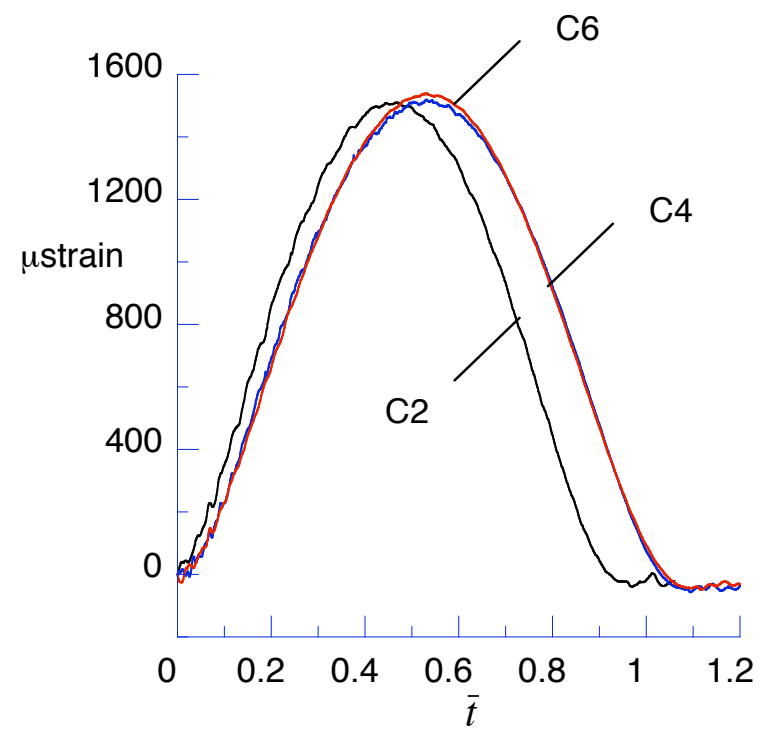

(b) Normalized and adjusted axial strain profiles

Figure 8. Experimental contact force and axial strain profiles for the curved panel configurations centrally impacted at nominal $0.35 \mathrm{ft}-\mathrm{lb}$ and $2.8 \mathrm{ft}-\mathrm{lb}$ impact energy for the reference and scaled-up configurations, respectively. 


\section{Experimental Results - Damage-Inducing Impact}

Normalized and adjusted experimental contact force profiles for the flat and curved panel configurations for the lower damage-inducing impact energy level are shown in Figs. 9a and b, respectively. The impact tests were performed at a nominal impact energy level of $1.125 \mathrm{ft}-\mathrm{lb}$ for the reference panels, and at a nominal impact energy level of $9.0 \mathrm{ft}-\mathrm{lb}$ for the scaled-up panels. The scaled contact force profiles for the flat panel configurations shown in Fig. 9a agree well, in terms of peak force, contact duration, and the overall shape of the curves, even when damage is present. Similar results are shown for the curved panels in Fig. 9 b.

The threshold for damage initiation, as well as the manifestation of the damage, differs significantly for the three configurations. For the panel configurations tested, the point at which damage initiates is difficult to determine from the force profiles recorded from the impact tests. Static-indentation curves can be used to obtain some insight. As discussed previously, the load-displacement curves from the static indentation tests, shown in Fig. 2, indicate that there are no drops in the load-displacement curves for the flat and curved 8-ply laminates prior to reaching the ultimate load. For the sub-laminate scaled laminate, on the other hand, there is an indication of damage in the load response early in the loading, and for all of the scaled-up laminates ultimate load is preceded by at least one sharp drop in the load-deflection curve. The back surface strain profiles for the flat and curved panel configurations, shown in Figs. 10a and b, respectively, also indicate differences in the damage development between the configurations. The strain responses shown in Fig. 10a for the flat panel configurations suggest that more significant damage is developing in the ply-level scaled panel than in the reference and sub-laminate scaled panel. The large strain response for the sub-laminate scaled panel compared to the other two panels is possibly the result of the impactor striking the panel off center, and closer to the gage. The strain responses shown in Fig. 10b for the curved panel configurations indicate, on the other hand that more damage is developing in the sub-laminate scaled panel than in the reference and ply-level scaled panels. The maximum strain magnitude for the reference panel exceeded the maximum range set in the test, so the data is truncated.

Similar observations regarding the damage in flat and curved panels were made by C-scan inspection and deplying. Representative $\mathrm{C}$-scan images of the flat panels after the $1.125 \mathrm{ft}-\mathrm{lb}$ impact for the reference panel and after the $9.0 \mathrm{ft}-\mathrm{lb}$ impact for the scaled panels are shown in Fig. 11. The projected damage area for the reference 8-ply flat and curved panels is very small and elliptical in shape, and no fiber breakage is visible after de-plying. The projected damage area for the sub-laminate scaled panels is nearly circular in shape, and approximately six times larger than the damage area for the reference configuration, and also free of fiber breakage on inspection after deplying. In the case of the flat, ply-level scaled laminates, the projected damage area is elliptical in shape and is much larger (approximately 17 times) than the damage area for the reference configuration. In addition, localized fiber

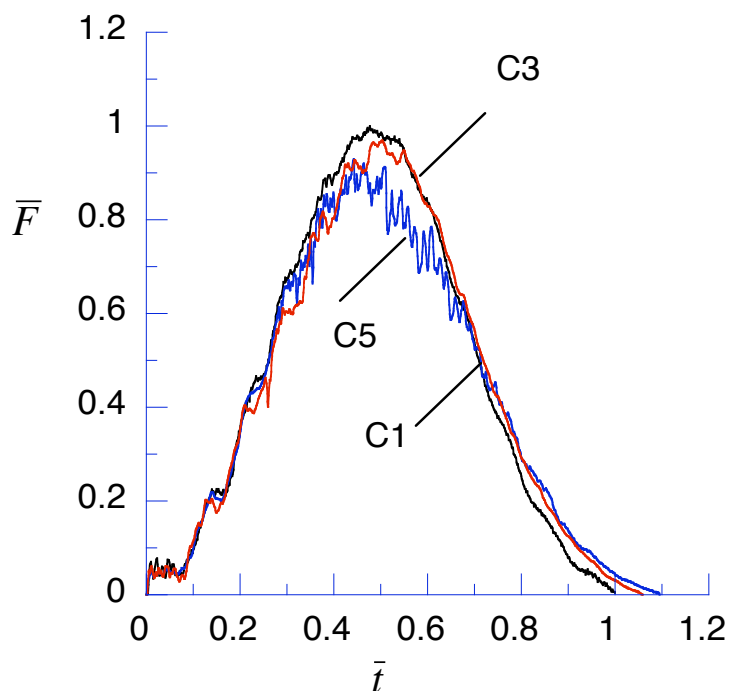

(a) Flat Panels

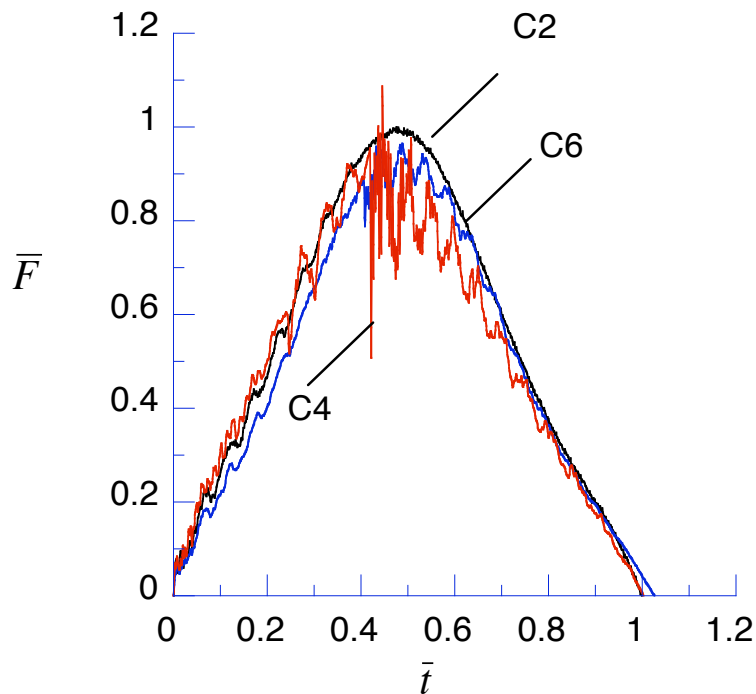

(b) Curved Panels

Figure 9. Experimental normalized and adjusted contact-force profiles for the flat and curved panel configurations impacted at $\mathbf{1 . 1 2 5} \mathrm{ft}-\mathrm{lb}$ and $\mathbf{9 . 0} \mathrm{ft}-\mathrm{lb}$ nominal impact energy for the reference and scaled configurations, respectively. 


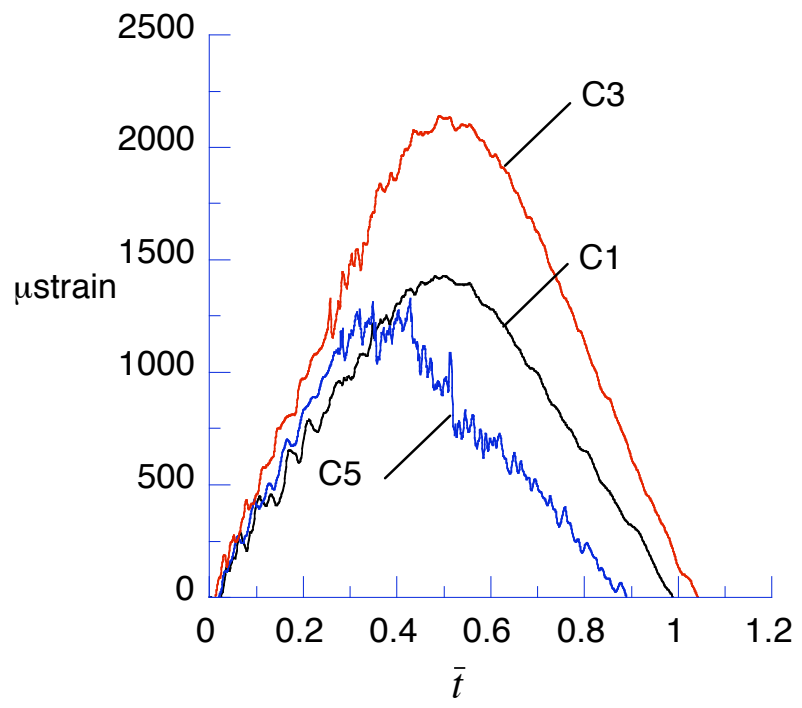

(a) Flat Panels

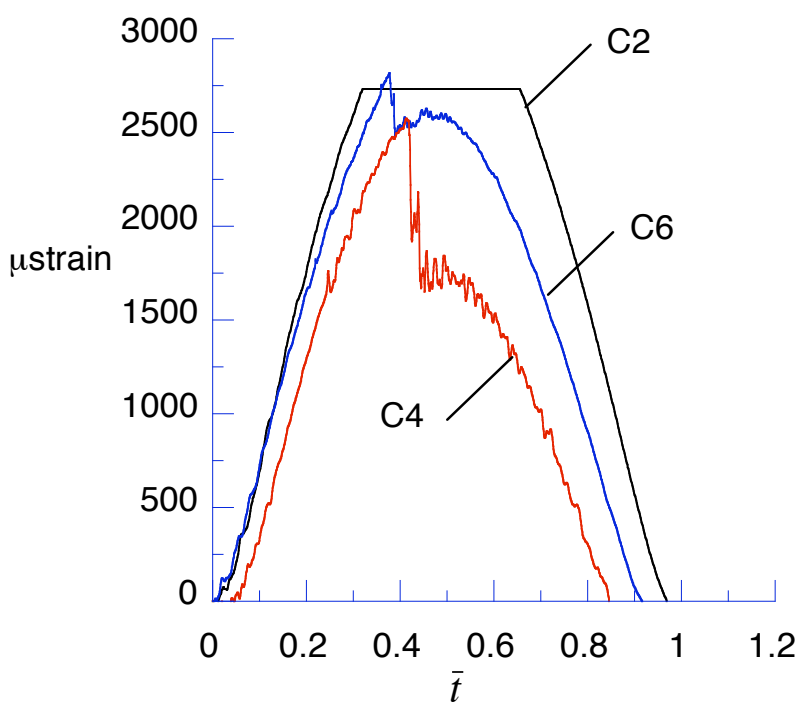

(b) Curved Panels

Figure 10. Experimental normalized and adjusted strain profiles for the flat and curved panel configurations impacted at $1.125 \mathrm{ft}-\mathrm{lb}$ and $9.0 \mathrm{ft}-\mathrm{lb}$ nominal impact energy for the reference and scaled configurations, respectively.

breakage was observed in every ply. The difference in damage mechanisms and extent between the ply-level and sub-laminate scaled flat panels suggests that for the $9 \mathrm{ft}-\mathrm{lb}$ impact energy, the ply-level scaled laminate is less damage resistant than the sub-laminate scaled laminate.

Contact force profiles for the flat and curved panels configurations for the higher damage-inducing impact energy level are shown in Figs. 12a and b, respectively. The impact tests were performed at a nominal impact energy level of $2 \mathrm{ft}-\mathrm{lb}$ for the reference panels, and at a nominal impact energy level of $16.0 \mathrm{ft}-\mathrm{lb}$ for the scaled-up panels. Even though at first glance the peak force of the reference and scaled configurations shown in Fig. 12 appear to be nearly equal, a closer look at the results in Fig. 12 shows that the contact duration for the reference configuration is shorter than the contact duration for the scaled configurations, indicating that more extensive damage has developed in the scaled configurations. The experimental strain profiles are shown in Figs. 13a and b. For both the flat and curved panel configurations, the damage in the form of loss of stiffness occurs at the same normalized time. The strain profiles indicate that the loss of stiffness in the reference configuration and the sublaminate scaled configuration are similar, indicating similar extent of fiber damage. For the ply-level scaled configuration, the loss of stiffness occurs earlier. In all three cases the fiber damage is extensive and no comparison of contact durations can be made. The results for the curved configurations in Fig. 13b suggest that the damage in the curved configurations is less extensive than the damage in the flat configurations. In addition, the curved reference configuration appears to have less damage than the scaled configurations. Furthermore, the results suggest that the sub-laminate scaled flat and curved panels have more extensive damage than the ply-level scaled flat and curved panels.

C-scan projections of the damaged areas in the panels subjected to the higher damage-inducing impact energy show trends similar to those described above for the panels subjected to the lower damage-inducing impact energy. In addition, extensive back surface damage with fiber breakage coming through the back surface was observed on the sub-laminate scaled flat panel. Only back surface splits were observed on the ply-level scaled flat panel. It appears as though the incident energy of the impactor is dissipated in delamination damage mechanisms in the plylevel scaled laminate, since the stacked ply laminate is more prone to delamination damage. The sub-laminate scaled flat panel, on the other hand appears to absorb the incident energy through localized fiber breakage, and has more significant damage than the ply-level scaled flat panel at the higher impact energy.

The difference described above in damage extent and damage mechanisms in the reference and the scaled configurations suggest that the projected damage area, and the extent of fiber breakage do not scale according to the scaling relationships provided in Table 1. For both of the impact energies that induced damage, the scaled flat and curved laminates appear to develop more damage than the reference configuration. The results also show that even though significant differences exist between the damage states in the three configurations impacted at the lower 


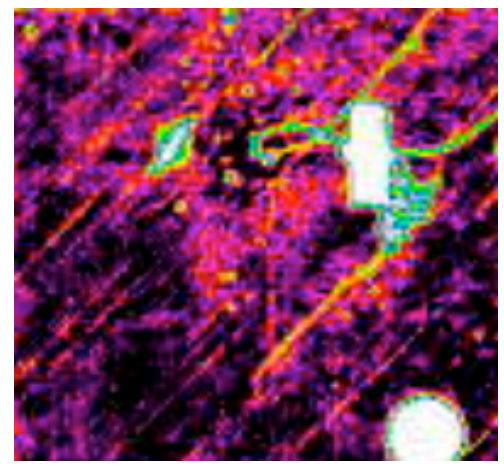

(a) 5 in. $X 5$ in., $[45 / 0 /-45 / 90]_{s}$

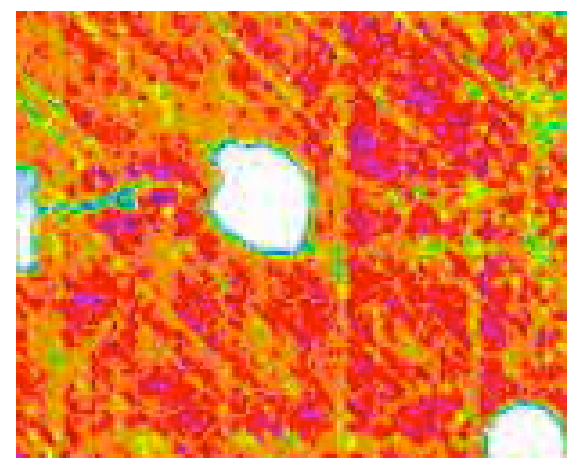

(b) 10 in. $X 10$ in., $[45 / 0 /-45 / 90]_{2 s}$

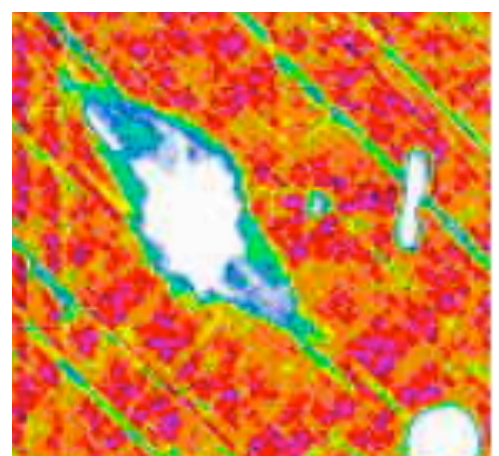

(c) 10 in. $X 10$ in., $\left[45_{2} / \mathbf{O}_{2} /-45_{2} / 90_{2}\right]_{s}$

Figure 11. C-scan damage images for the 3 flat configurations subjected to $1.125 \mathrm{ft}$-lb and $9.0 \mathrm{ft}-\mathrm{lb}$ nominal impact energy for the reference and scaled configurations, respectively.

Damage-inducing energy, the contact force profiles scale. These results indicate that for the panels considered in the present study, the value of the peak force from a force-time profile is not sufficient as a damage metric or to perform comparisons across structural configurations.

For structures exhibiting geometrically linear response, the residual stiffness (the ratio of pristine to damaged contact durations) and the relative coefficient of restitution loss have been employed to quantify the loss in structural stiffness due to the onset of damage [19]. Coefficient of restitution and residual stiffness plots are shown in Figs. $14 \mathrm{a}$ and $\mathrm{b}$, respectively for the flat and curved panel configurations considered in this study. The curves show that the reference 8-ply flat panel and the sub-laminate scaled flat panel exhibit a sharp increase in damage extent, indicated by the sharp decrease in COR, and a sharp decrease in residual performance with increase in impact energy. The results for the ply-level scaled laminate, on the other hand, indicate a more progressive damage accumulation for this configuration than for the other configurations. Consequently, the results indicate that at lower impact energies, the sub-laminate scaled flat panel configuration has more residual stiffness, and at higher impact energies, the ply-laminate scaled flat panel

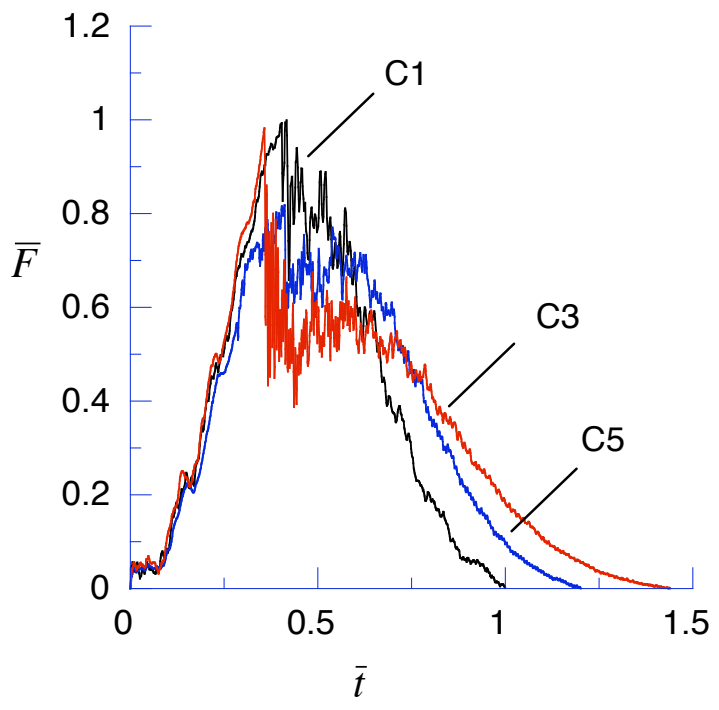

(a) Flat panels

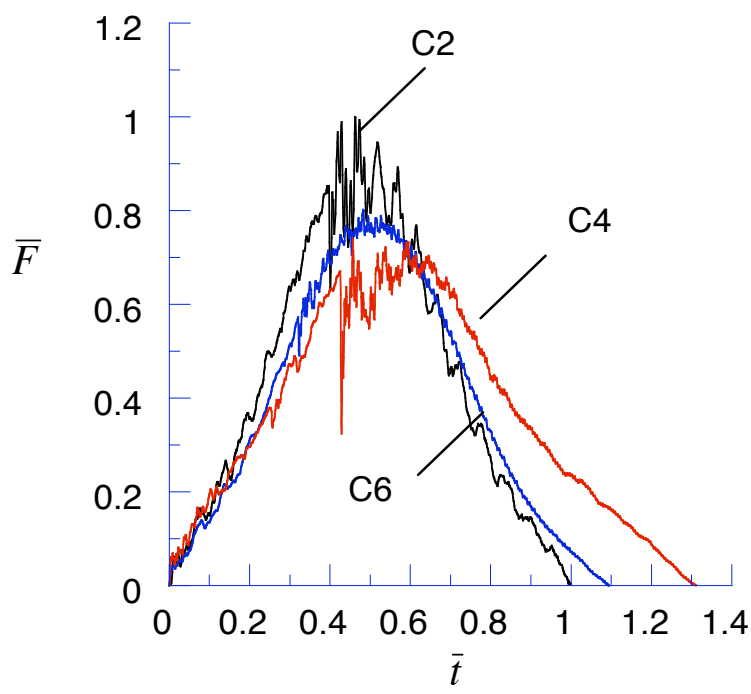

(b) Curved panels

Figure 12. Experimental adjusted contact-force profiles for the flat and curved configurations impacted at 2.0 ft-lb and 16.0 ft-lb nominal impact energy for the reference and scaled configurations, respectively. 
configuration has more residual stiffness. More data are needed between the low and high damage-inducing impact energies to verify this trend. The curves for the curved panel configurations indicate that the ply-level scaled laminate has greater residual stiffness than the sub-laminate scaled laminate for all of the impact energies tested. In addition, the damage extent in the curved panel configurations levels off, compared to the flat panel configurations, with increase in impact energy. This trend is consistent with previous observations $[8,11]$ indicating that in the curved panels some of the incident energy is likely dissipated in large structural deformation rather than in damage creation. In addition, if the damage scaled between the reference and scaled configurations, both the COR and the residual stiffness would be equal for 8- and 16-ply configurations. It is evident from Fig. 14, that they are not equal for the 8-and 16-ply configurations. However, the general trend of both quantities is the same for the reference and scaled configurations.

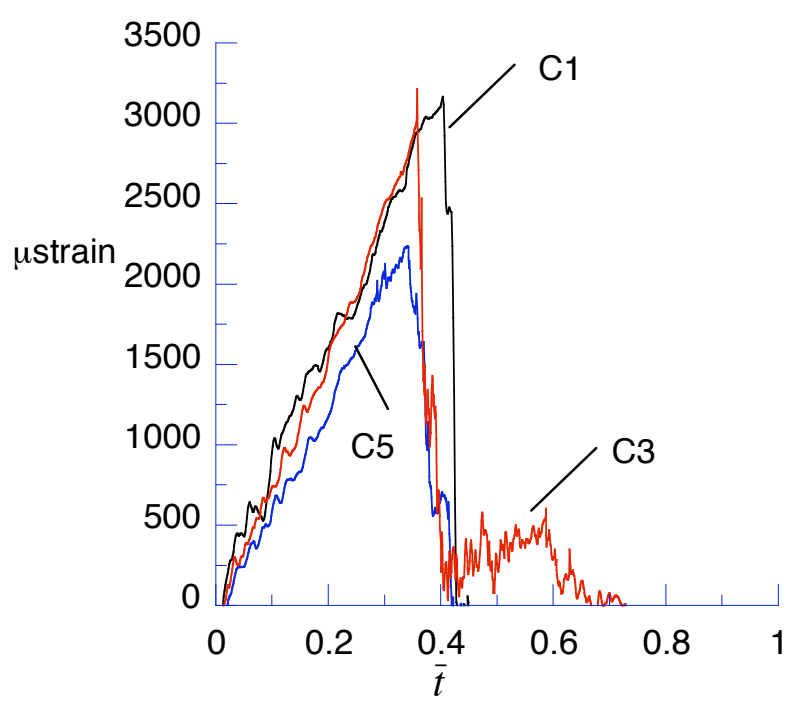

(a) Flat panels

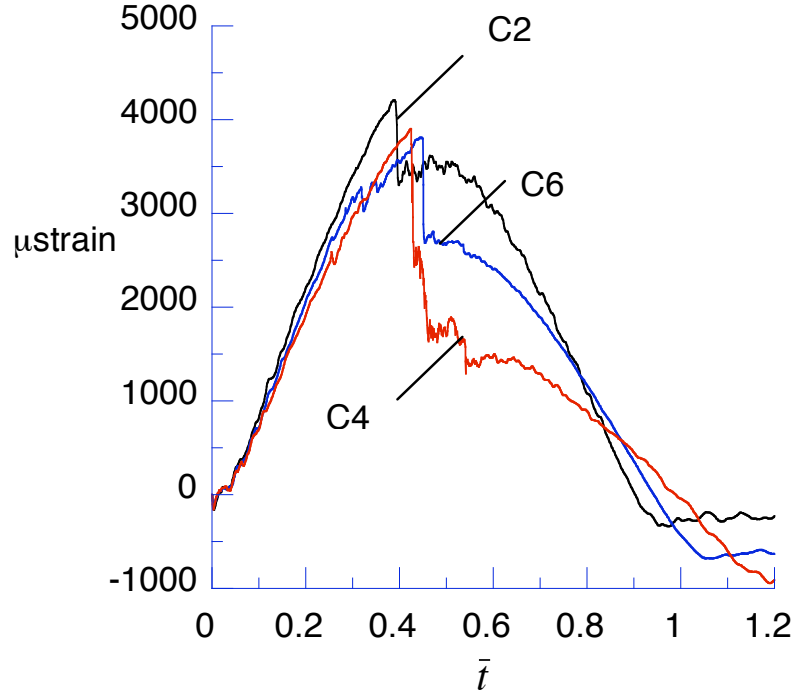

(b) Curved panels

Figure 13. Experimental and normalized adjusted strain profiles for the flat and curved configurations impacted at $2.0 \mathrm{ft}-\mathrm{lb}$ and $16.0 \mathrm{ft}-\mathrm{lb}$ nominal impact energy for the reference and scaled configurations, respectively.

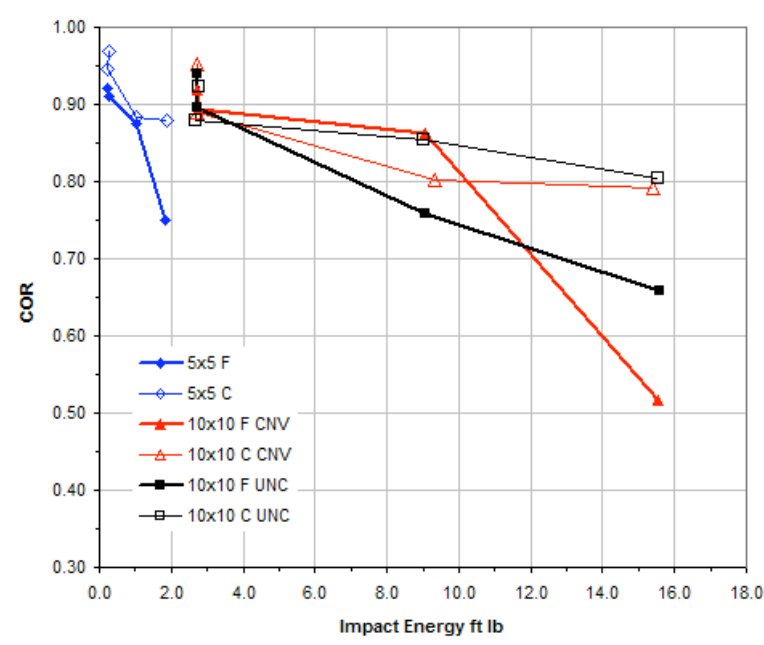

(a) COR

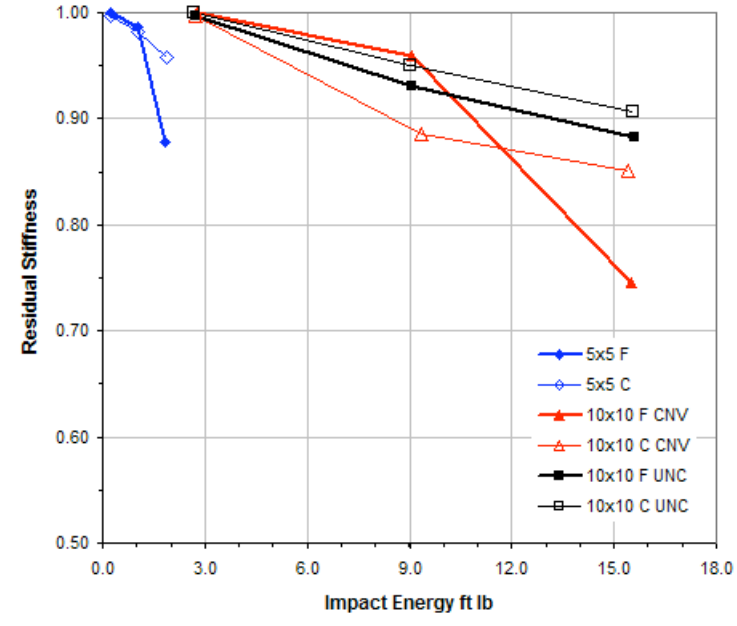

(b) Residual stiffness

Figure 14. Effect of impact energy on the COR and panel residual stiffness for the 3 flat and 3 curved panel configurations. 


\section{Discussion and Conclusions}

The results of a preliminary analytical and experimental study to determine the accuracy of scaling laws for thin, flat and curved panels exhibiting geometrically non-linear response when subjected to low-velocity transverse impact have been presented. It has been shown in the case of cylindrically curved panels the radius of the panel is a geometric scalable parameter. The effect of damage on the scalability of the structural response characteristics, and the effect of scale on damage development are also presented. Assessment of the scaling laws is made for two sizes of flat and curved panels that have been geometrically scaled. Thickness scaling is accomplished using ply-level and sublaminate scaling for scaled flat and curved configurations. Both elastic impact and two levels of damageinducing impact energies are considered. The lower damage-inducing impact energy is high enough to cause delamination and other matrix damage, and the higher damage-inducing impact energy is high enough to cause extensive fiber damage. Damage is evaluated using conventional methods, including C-scan, specimen de-plying and visual inspection of the impacted panel. Coefficient of restitution and normalized contact duration (residual stiffness) are also used to assess the extent of damage.

The analysis and experimental results show that, for elastic impacts, the overall geometrically nonlinear structural response of the panels, as indicated by contact force vs. time and axial strain vs. time profiles, scales according to the scaling laws. Similarly, the scaled contact force profiles agree well, in terms of peak force, contact duration, and the overall shape of the curves, for the lower energy damage-inducing impact. The threshold for damage initiation, as well as the manifestation of the damage, differs significantly, however, for the configurations considered. These results indicate that peak impact force alone is not sufficient as a damage metric or to compare across configurations. At the higher damage-inducing impact energy, the difference between the reference and scaled configurations is evident and the contact-force profiles do not scale. The contact force profiles, however, do not provide an adequate indication of the damage accumulated. When possible, C-scan or X-ray investigations should be complemented by destructive inspection methods, such as resin burn-off and de-plying to identify the different failure mechanisms. The ratio of pristine to damaged contact duration (residual stiffness), and the Coefficient of Restitution (COR), decreased as the severity of damage increased, but did not scale.

From the impact and static indentation test results presented it appears as though there is a size effect that influences the damage mode and extent in the scaled laminates. For both of the damage-inducing impact energies, the scaled flat and curved laminates appear to develop more severe damage than the reference configuration. In addition, for the panels studied in this investigation, the relative contribution of delamination failures and fiber failures to the impact energy absorption appears to be dependent on the approach used to scale the laminate, i.e., plylevel or sub-laminate scaling, the panel radius, and the impact energy. For the flat panels subjected to the lower damage-inducing impact energy, the ply-level scaled laminate appears to be less damage resistant than the sublaminate scaled laminate, exhibiting larger projected delamination area than the sub-laminate scaled laminate, and fiber breakage. For the flat panels subjected to the higher damage-inducing impact energy, the ply-level scaled laminate appears to be more damage resistant, absorbing more energy through delamination damage than fiber failure, than the sub-laminate scaled laminate, which appears to absorb more energy through local fiber failures. For the curved panels, on the other hand, the sub-laminate scaled laminate appears to be less damage resistant than the ply-level scaled laminate. In addition, curved panels dissipate energy in structural deformation and retain higher relative residual stiffness than the corresponding flat panels.

The results indicate that at lower impact energies, the sub-laminate scaled flat panel configuration has more residual stiffness, and at higher impact energies, the ply-laminate scaled flat panel configuration has more residual stiffness. Further investigation needs to be carried out at intermediate impact energies between the low and high damage-inducing impact energies to verify this trend.

\section{References}

${ }^{1}$ Abrate, S. "Impact on Laminated Composite Materials," Applied Mech. Rev., Vol. 44, No. 4, April 1991, pp. 155-189.

${ }^{2}$ Cantwell, W. J., and Morton, J., "The Impact Resistance of Composite Materials - A Review," Composites, Vol. 22, No. 5,September 1991, pp. 347-362.

${ }^{3}$ Abrate, S., Impact on Composite Structures, Cambridge University Press, 1998.

${ }^{4}$ Cantwell, W. J., "The Influence of Target Geometry on the High Velocity Impact Response of CFRP," Composite Structures, Vol. 10, 1988, pp. 247-265.

${ }^{5}$ Cantwell, W. J., and Morton, J., "Geometrical Effects in the Low Velocity Impact Response of CFRP," Composite Structures, 1989, pp. 39-59.

${ }^{6}$ Ambur, D.R., Starnes, J. H. Jr., "Influence of Large Deflection Effects on the Impact Response of Flat and Curved Composite Plates", 36 ${ }^{\text {th }}$ AIAA Structures, Structural Dynamics and Materials Conference, No. 95-1205, April 1995. 
${ }^{7}$ Ambur, D. R., Starnes, J. H. Jr., "Nonlinear Response and Damage Initiation Characteristics of Composite Curved Plates Subjected to Low-Speed Impact", $38^{\text {th }}$ AIAA Structures, Structural Dynamics and Materials Conference, No. 97-1058, April 1997.

${ }^{8}$ Ambur, D. R, Starnes, J. H. Jr., "Effect of Curvature on the Impact Damage Characteristics and Residual Strength of Composite Plates", $39^{\text {th }}$ AIAA Structures, Structural Dynamics and Materials Conference, No. 98-1881, April 1998.

${ }^{9}$ Ambur, D. R, Starnes, J. H. Jr., Prasad C. B., "Influence of Target and Impactor Parameters on the Nonlinear Response and Residual Strength of Curved Thin Composite Plates", $41^{\text {st }}$ AIAA Structures, Structural Dynamics and Materials Conference, No. 2000-1591, April 2000.

${ }^{10}$ Kistler, L. S., Waas, A. M., "Impact Response of Cylindrically Curved Laminates Including A Large Deformation Scaling Study", International Journal of Impact Engineering, Vol. 21, 1-2, 1998, pp. 61-75.

${ }^{11}$ Wardle, B. L., and Lagace, P. A., "Importance of Instability in Impact Response and Damage Resistance of Composite Shells," AIAA Journal, Vol. 35, No. 2, Feb., 1997.

${ }^{12}$ Qian, Y., Swanson, S. R., Nuismer, R. J., Bucinell, R. B., “An Experimental Study of Scaling Rules for Impact Damage in Fiber Composites," Journal of Composite Materials, Vol. 24, May 1990, pp. 559-570.

${ }^{13}$ Sankar, B. V., "Scaling of Low-Velocity Impact for Symmetric Composite Laminates," Journal of Reinforced Plastics and Composites, Vol. 11, 1992, pp. 297-305.

${ }^{14}$ Nettles, A.T., Douglas, M.J., Estes, E. E., "Scaling effects in carbon/epoxy laminates under transverse quasi-static loading," NASA TM 209103, March 1999.

${ }^{15}$ Liu, D., Raju, B.B., Dang, X., "Size Effects on Impact Response of Composite Laminates,” Journal of Impact Engineering, Vol. 21, No. 10, 1998, pp. 837-854.

${ }^{16}$ Morton, J., "Scaling of Impact Loaded Carbon Fiber Composites", AIAA Structures, Structural Dynamics and Materials Conference, No. 87-0867, 1987, pp. 819-826.

${ }^{17}$ Swanson, S. R., Smith, N. L., and Qian, Y., "Analytical and Experimental Strain Response in Impact of Composite Cylinders," Composite Structures, Vol. 18, 1991, pp. 95-108.

${ }^{18}$ Swanson, S. R., "Scaling of Impact Damage in Fiber Composites from Laboratory Specimens to Structures," Composite Structures, 1993, pp. 249-255.

${ }^{19}$ Feraboli, P., Kedward, K. T., "Enhanced Evaluation of the Low-Velocity Impact Response of Composite Plates", AIAA Journal, 42/10, October 2004, pp. 2143-2152.

${ }^{20}$ Dugundji, J., "Simple Expressions for Higher Vibration Modes of Uniform Euler Beams," AIAA Journal, Vol. 26, No. 8, August 1988, pp. 1013-1014.

${ }^{21}$ Kwon, Y. S., and Sankar, B. V., "Indentation-Flexure and Low-Velocity Impact Damage in Graphite Epoxy Laminates," Journal of Composites Technology and Research, Vol. 15, No. 2, 1993, pp. 101-111. 